\title{
Effects of Radiosynovectomy with P-32 Colloid Therapy in Hemophilia and Rheumatoid Arthritis
}

\author{
Victoria Ester Soroa, ${ }^{1,2}$ Mańa del Huerto Velázquez Espeche, ${ }^{1}$ Carlos Giannone, ${ }^{1}$ \\ Horacio Caviglia, ${ }^{3}$ Gustavo Galatros, ${ }^{3}$ Daniel Fernández, ${ }^{2}$ Marcos Méndez, ${ }^{2}$ \\ Gustavo Guillermo Naswetter, ${ }^{2}$ and Jorge Osvaldo Nicolini ${ }^{4}$ \\ ${ }^{1}$ Centro de Medicina Nuclear, Comision Nacional de Energía Atómica Argentina, Buenos Aires, \\ IAEA, Argentina \\ ${ }^{2}$ Rheumatology, Hospital De Clínicas "José de San Martin," Universidad de Buenos Aires, Buenos \\ Aires, Argentina \\ ${ }^{3}$ Traumatology and Orthopedics, Hospital Municipal, J. A. Fernández, Hemophilic Foundation, Buenos \\ Aires, Argentina \\ ${ }^{4}$ Radiopharmacy, Laboratory Bacon, Buenos Aires, Argentina
}

\begin{abstract}
Aim: The aim of this study was to assess the effects of treatment with our locally produced P-32 colloidal suspension on knee synovitic inflammations of hemophilic and rheumatoid arthritis (RA) patients, as well as to compare results with chemical synovectomy or corticoid intra-articular injections and evaluate the cost-benefit ratio. Materials and Methods: Thirty-six hemophilic male patients, 4-28 years of age and sent by the Hemophilic Foundation (Buenos Aires, Argentina), were enrolled for knee radiosynovectomy (RS) with P-32 colloid (26 patients), or the antibiotic rifampicin with the cooperation of orthopaedists (10 patients). Parents' informed consent was obtained. The following procedures were performed: routine blood tests, X-ray, ultrasound, a 3-phase bone scan, plus monthly methylene diphosphonate (MDP) controls. Patients were included in this study only if several knee episodes had occurred. Exclusion criteria included bone destruction and big Baker's cyst. Twelve RA patients were included, with similar selection criteria: 6 RA patients received P-32 therapy, and the other 6 patients intra-articular corticoids. Clinical, blind evaluation (state of joint involvement, pain, motility, requirements of antihemophilic factors, corticoids, or analgesics) was registered in follow-up charts. If required, joint aspiration was carried out. Intra-articular instillation of saline plus flushing was done before the needle was withdrawn. $P$ 32 Bremsstrahlung emission was used in the gamma camera for early and late imaging to confirm the absence of leakage. For intra-articular chemical injections therapy, $4 \mathrm{MBq}$ of Tc-99m MAA (macroaggregates) was used. Immobilization and relative rest for 72 hours followed the procedures. Results: There were neither local or systemic effects, nor leakage during P-32 treatment. Intra-articular rifampicin and corticoids procedures required frequent injections. Comparison of regions of interest (ROIs) in treated knees during soft-tissue scintigraphies in pre- and post-third MDP control showed knee improvement. The follow-up evaluation demonstrated an increase in joint motion, diminished volume, and less requirement and frequency of the use of antihemophilic factors (AHF) in 80\% of the radiosynovectomies (21 of 26), thus lowering health costs. Five female RA patients (5 of 6) had decreased joint swelling and pains, resulting in increased joint motion. Conclusions: Radiosynovectomy in RA showed a 3-month pain pallia-
\end{abstract}

Address reprint requests to: Victoria E. Soroa; Centro de Medicina Nuclear, Hospital De Clínicas "José de San Martín," Universidad de Buenos Aires, Radiobiología, Comisión Nacional de Energía Atómica; Avenue del Libertador 8250, CP 1429, Buenos Aires, Argentina; Tel.:/Fax: \#541148052123

E-mail: soroa@cnea.gov.ar 
tive effect. One intra-articular knee radiosynoviorthesis in haemophilic patients provides a more than 3 month relief of symptoms after treatment with locally produced P-32 (11 patients). This turned out to be a safe, economic alternative procedure in emerging nations where the availability of AHF is difficult and expensive.

Key words: radiosynovectomy, P-32 colloid, hemophilia, rheumatoid arthritis

\section{INTRODUCTION}

Radiosynovectomy (RS) induced by an intra-articular injection of beta-emitter suspension, is a local therapeutic tool used in the treatment of hemartrosis and rheumatoid arthritis (RA), ameliorating synovial inflammation.

Several intrarticular beta emitters are approved in Europe for the treatment of painful synovial joint pathologies: ${ }^{1,2}$ 90-Y silicate/citrate, 186-Re sulphide, and 169-Er citrate. Others are under investigation, such as 188-Re, 165-Dy, and 166Ho. Depending on the physical characteristics of the beta radiopharmaceutical will be the indication to treat different types of joints. The therapeutic team must study the patient jointly with the orthopaedic of the hemophilic patients and the rheumatologist. All members of the therapeutic team must be trained in the use of unsealed source therapy.

$\mathrm{RA}$ is a systemic disease and is to be treated accordingly. Local intra-articular treatment of the affected joints, such as chemical, radiochemical, and surgical synovectomy, are applied after proper evaluation of risk and patient's benefit.

Haemophilia is a worldwide disease affecting males, including young children; management of the associated arthropathy is often compromised in emerging countries by high cost (injection of antihemophilic factors (AHF) and limited availability of specialized treatments.

Treatments are aimed to provide pain relief and improved joint mobility. Yet, progression of the disease continues. Surgical synovectomy is expensive, requires hospitalization, and is limited to a maximum of 3 years. Chemical synovectomy damages cartilage and bones. RS offers an attractive alternative for the effective management of synovitis in the early stages of the disease, in order to control synoviocytes proliferation. Earlier attempts with this therapy were tried several years ago, although with limited success. The radiocolloids, such as ${ }^{198} \mathrm{Au}$ and ${ }^{32} \mathrm{P}$, showed excessive leakage from the joint. New developments of colloid particles, such as hydroxide, citrates, silicates, or macroaggregates (MAA) with appropriate size that labels different beta emitters, diminished the unwanted joint leakage and the radiation exposure to different organs. ${ }^{3,4}$

Waste and disposal of used materials must also be carefully handled.

\section{Characteristics of a Radiocolloid for RS}

Characteristics of a radiocolloid for RS were a short half-life, penetration of $\beta^{-}$emissions of adequate energy to be used in a certain joint and ablate the proliferating synovium with minimal radiation dose to the underlying cartilage and bones, radio chemical stability, and a size range of $150-200 \mathrm{~nm}$ (to be phagocytosized without signs of inflammation). ${ }^{1,5-7}$

The Atomic Energy Agency (IAEA) established a Coordinated Research Project on Comparative Evaluation of the Efficacy of Radiosynovectomy with Conventional Intra-articular Therapy in Rheumatoid Arthritis and Haemophilic Arthropathy, ${ }^{8,9}$ by which 12 countries are participating in this study, among them Argentina. Our local availability is P-32-enriched gelatine colloid, which we applied only in knees of the above-mentioned pathologies.

\section{Exclusion Criteria}

Joint instability, bone destruction, grade IV osteorthritis, big Baker's cyst, skin or joint infection, less than 2 years for haemophilia, present acute episodes of bleeding at the time of injection.

\section{Pregnancy in rheumatoid arthritic (RA) patients}

Patients' or parents' had to provide informed consent obtained prior to enrollment.

\section{MATERIALS AND METHODS}

Characteristics of P-32: maximum beta range $1.71 \mathrm{MeV}$, maximum tissue penetration $7.9 \mathrm{~mm}$, 
mean penetration $2.2 \mathrm{~mm}$, half-life in days, 14.3. Injected activity in adults was 11-74 MBq.

We were provided with a sterile P-32-enriched gelatine chromic phosphate colloid with a size of 100-200 nm by our local radiopharmacist company (Laboratorios Bacon S.A.I.C., Buenas Aires, Argentina).

Children 2-6 years of age received one-third of the adult activity, children 6-10 years of age used half activity, and children 10-16 years of age were given the $75 \%$ of the adult dose.

Thirty-six hemophilic male patients, 4-28 years of age were sent by the Hemophilic Foundation, (Buenos Aires, Argentina) were enrolled for knee radiosynovectomy with P-32 colloid (26 patients), or the antibiotic rifampicin with the cooperation of orthopaedists (10 patients).

Twelve RA patients, 30-60 years of age, were included; 6 patients received P-32 therapy and the other 6 patients intra-articular corticoids. Systemic medication had to be maintained.

To the other chemical synovectomies with which we compared treatment results we added $4 \mathrm{MBq}$ of MAA $99 \mathrm{~m}-\mathrm{Tc}$ in order to be able to blind the evaluating team of which patients received radiotherapeutic treatment.

Documentation of patient history using a standard set of questions in Case Report Forms included age, diagnosis RA or Hemophilia (A or B), severity of hemophilia, previous AHF therapy, number of episodes of hemartrosis, pain (visual analog scale in 10 steps), joint swelling (circumference of joint), range of motion, and walking ability. Complimentary laboratory screening for blood tests, X-ray, ultrasound, clinical evaluation, a 3-Phase MDP scan pre-P-32 was carried out in a semiquantitave fashion.

\section{Protocol for Hemophilia and RA}

The procedure was undertaken in a clean room. Intra-articular injection was performed with sterile instruments. Unit dosis of the P-32 phosphate chromic colloid was drawn in a laminar flow hood. The intra-articular dose was in accordance to patients' age, joint (volume), and body weight. Synovial or hematic content was previously evacuated from the compromised joint. The injection was performed under gamma-camera control. We could only treat knee joints because of the characteristics of P-32 colloid. 7,8 After the radiopharmaceutical injection, the needle was flushed with $1 \mathrm{cc}$ of saline. The joint was manipulated through a full range of motion to distribute the radiocolloid throughout the joint space. ${ }^{1,10}$

Post-therapeutic procedure gamma-camera knee images and whole-body scans were obtained at 24-72 hours to document the distribution of radiopharmaceutical in the joint and to confirm the absence of leakage. For P-32, we set our gamma camera to acquire the "Bremsstrahlung" emission, in 201-Tl photo peak settings with a $25 \%$ window.

We immobilized the injected joint by splinting for 2 days.

\section{Follow-Up}

The follow-up consisted of a routine history and clinical examination with the same set of questions as before treatment, registering the number of subsequent episodes, range of movement, joint circumference, and the amount of AHF requirements. MDP-scan evaluation on the 2-Phase at 1 , 3,6 , and 12 months where irregular, and similar regions of interest (ROIs) where drawn on the

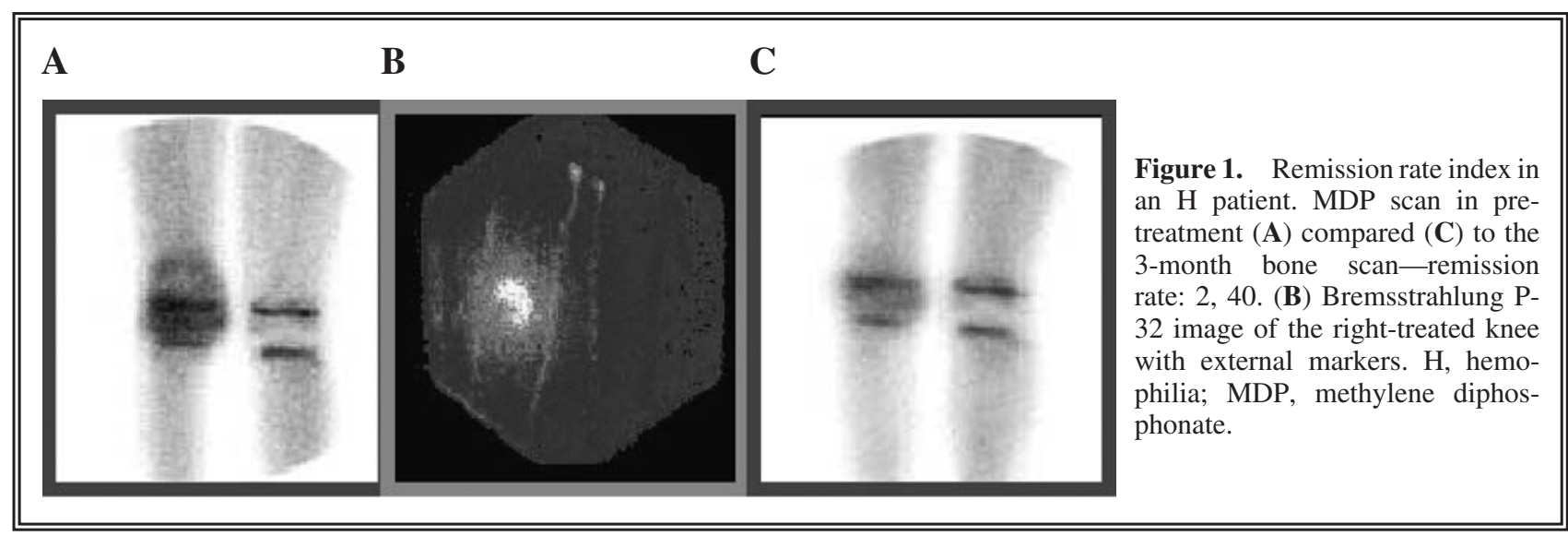




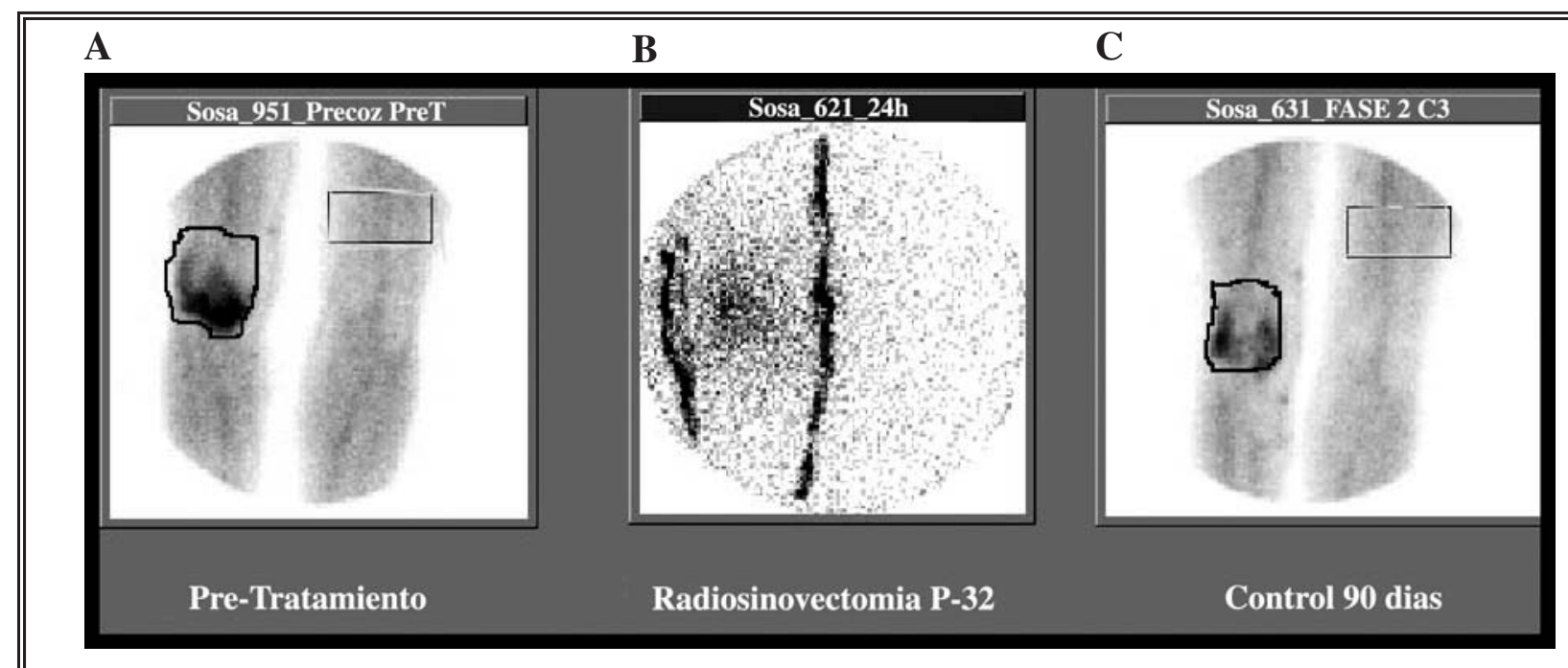

Figure 2. Remission rate index in a RA patient. MDP scan in pretreatment $(\mathbf{A})$ compared $(\mathbf{C})$ to the 3-month bone scan-remission rate: 1, 90. (B) Bremsstrahlung P-32 image of the right-treated knee with external markers. RA, rheumatoid arthritis; MDP, methylene diphosphonate.

treated knee and in a background area on the contralateral thigh. From these analyses we obtained a remission rate.

\section{RESULTS}

There were neither local nor systemic effects, nor was there leakage during P-32 treatment. Traditional intra-articular rifampicin and corticoid procedures require frequent injections. Comparison of ROIS ratios in treated knees during soft-tissue scintigraphy in pre- and post-third-MDP control are demonstrative of knee improvement. An example is shown in Figure 1 for an hemophilic patient and in Figure 2 for a patient with RA.

Taking into account background, density, counts, and the number of pixels of the pre-P32 treatment and the follow-up 3rd MDP scan, we applied the following formula:

$$
\text { Severity Index }(t)=[(\mathrm{dL}-\mathrm{d} \mathrm{B}) / \mathrm{dB})] \cdot \mathrm{N} \mathrm{px}
$$

Time $(\mathrm{t})=$ Baseline, month, 3, 6, 9

where $\mathrm{dL}$ is the count density at the lesion ROI and $\mathrm{dB}$ is a background count density assessed in the other leg. The volume of the lesion is N pixels.

Remission rate: Severity Index $(S I)(0) / S I(\mathrm{t})$ must be greater than 1 for therapy success, while an improvement of at least $25 \%$ is considered clinically relevant. The follow-up evaluation

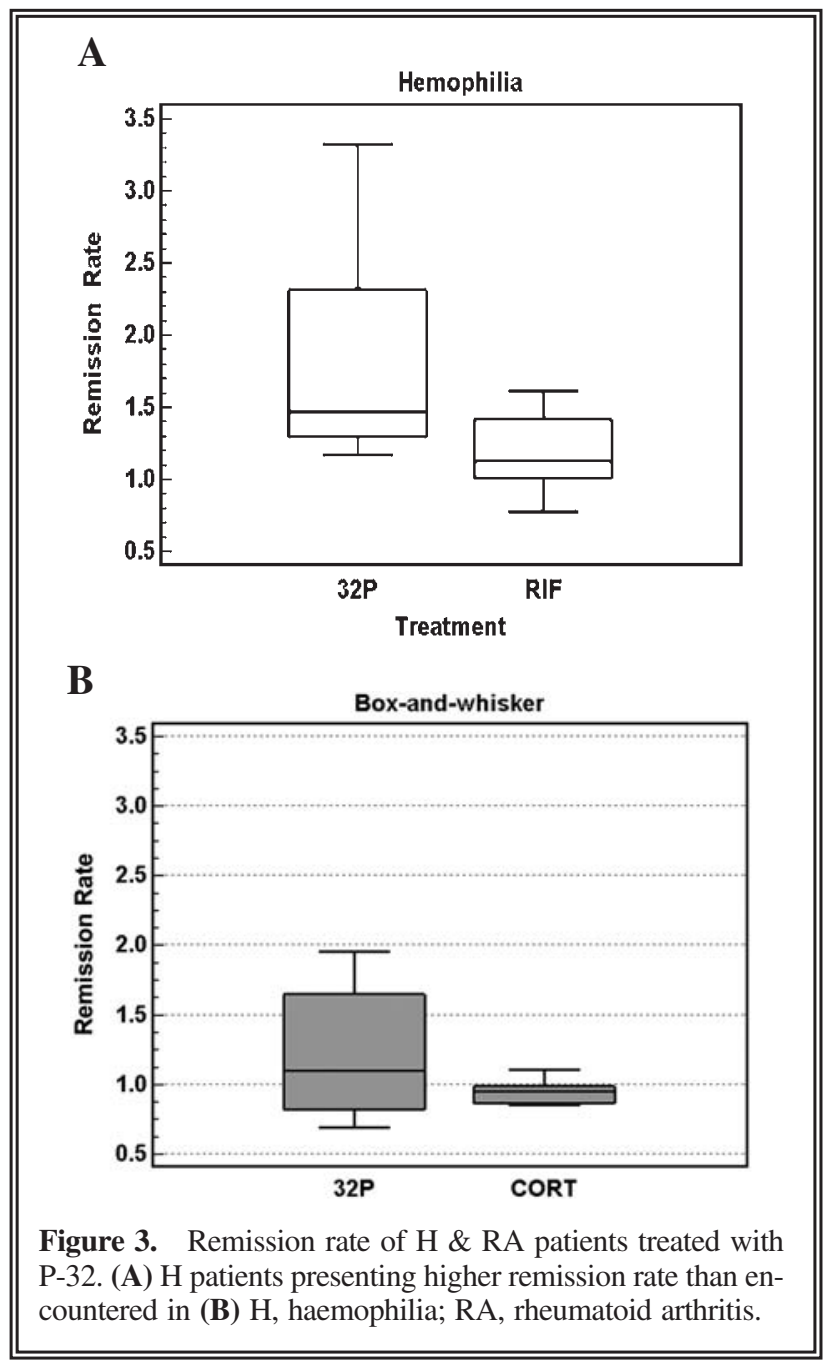


showed an increase in joint motion, diminished volume, and less requirement and frequency in the use of AHF in $80 \%$ of the radiosynovectomies (21 of 26 patients), thus lowering health costs. Figure $3 \mathrm{~A}$ illustrates this favorable outcome. The median remission rate for hemophilic patients treated with P-32 was 1.5 (range, 1.1-3.3), while the median for those that received rifampicin was $1.1(0.8-1.6) ; p<0.025$, (see Figure 3A).

Five RA female patients ( 5 of 6 patients) had similar joint improvements seen in Figure 3B.

\section{CONCLUSIONS}

Radiosynovectomy in RA showed a 3-month pain palliative effect. One intra-articular knee radiosynoviorthesis in haemophilic patients provides more than a 3-month relief of symptoms after treatment with locally produced P-32. ${ }^{11}$ The method has high potential as an alternative to the currently available modes of treatment. It turned out to be a safe, economic, alternative procedure in emerging nations, where availability of AHF is difficult and expensive.

\section{REFERENCES}

1. EANM Procedure Guidelines for Radiosynovectomy. Eur J Nucl Med 2003;30:12.
2. Clunie GPR, Ell PJ. A survey of radiation synovectomy in Europe, 1991-1993. Eur J Nucl Med 1995; 22:970.

3. Clunie GPR, Lovegrove FT. Radiation synovectomy. In: Ell PJ, Gambhir SS, eds. Nuclear Medicine in Clinical Diagnosis and Treatment, 3rd ed. Edinburgh: Churchill Livingstone, 1995:1051.

4. Farahati J, Reiners C, Fischer M, et al. Guidelines for radiosynoviorthesis [in German]. Nuklearmedizin 1999; 38:244.

5. Gobel D, Gratz S, v. Rothkirch T, et al. Radiosynoviorthesis with rhenium-186 in rheumatoid arthritis: A prospectivestudy of three treatment regimens. Rheumatol Int 1997;17:105.

6. Heuft-Dorenbusch LLJ, de Vet HCW, van der Linden $\mathrm{S}$. Yttrium radiosynoviortheses in the treatment of knee arthritis in rheumatoid arthritis: A systemic review. Ann Rheum Dis 2000;59:583.

7. Johnson LS, Yanch JC, Shortkroff S, et al. Beta-particle dosimetryin radiation synovectomy. Eur J Nucl Med 1995;22:977.

8. Jones G. Yttrium synovectomy: A meta-analysis of the literature. Aust N Z J Med 1993;23:272.

9. Fernandez-Palazzi F, Rivas S, Cibera JL, et al. Radioactive synoviorthesis in haemophilic haemarthrosis: Materials, techniques, and dangers. Clin Orthop 1996; 328:14.

10. Modder G. Radiosynoviorthesis: Involvement of $\mathrm{Nu}$ clear Medicinein Rheumatology and Orthopaedics. Meckenheim: Warlich, 1995.

11. Soroa VE, Velázquez Espeche MH, Giannone C, et al. Effects of radiosynovectomy P-32 colloid therapy in hemophilia and rheumatoid arthritis. [Abstr.] Eur J Nucl Med Mol Imaging 2004;31(Suppl 2):S477. 